\title{
Arcabouço de handoff definido por software e suporte à multihoming para redes veiculares heterogêneas
}

\author{
Henrique Júnior J. Pereira ${ }^{1}$, José Jailton H. F. Júnior ${ }^{2}$, Andson M. Balieiro ${ }^{1}$, \\ Tássio C. de Carvalho ${ }^{2}$, Kelvin L. Dias ${ }^{1}$ \\ ${ }^{1}$ Centro de Informática - Universidade Federal de Pernambuco (UFPE)
}

${ }^{2}$ Faculdade de Computação - Universidade Federal do Pará (UFPA)

\{hjjp, amb4, kld\}@cin.ufpe.br, josejailtonjunior@yahoo.com.br, tassio@ufpa.br

\begin{abstract}
Vehicle Ad Hoc networks (VANETs) allow passengers in smart vehicles to use multimedia applications, such as VoIP calls and video streaming. However, VANETS are dynamic networks, with vehicles moving frequently between the coverage cells of the access points, which can cause interruptions in the transmission of applications. Thus, effective handoff procedures to maintain the transmission while vehicles move around become imperative. This paper presents a handoff mechanism defined by software and with support for multihoming to guarantee seamless handoff in a heterogeneous vehicular scenario (IEEE 802.11p and LTE networks), ensuring the QoS/QoE levels required by the real-time application. The simulation results show the effectiveness of the proposed scheme when compared to the RSSI-based technique.
\end{abstract}

Resumo. As redes veiculares ad hoc (VANETs) possibilitam que passageiros de veículos inteligentes usufruam de aplicações multimídias, como chamadas VoIP e streaming de vídeo. Contudo, as VANETS são redes altamente dinâmicas, com veículos se movendo frequentemente entre as células de cobertura dos pontos de acesso, o que pode causar interrupções no fornecimento das aplicações. Dessa forma, procedimentos efetivos de handoff para manter a continuidade do fornecimento de conteúdo enquanto os veículos se locomovem se tornam imperativos. Este artigo apresenta um mecanismo de handoff definido por software e com suporte à multihoming para garantir o handoff transparente em um cenário veicular heterogêneo (Redes IEEE 802.11p e LTE), assegurando os níveis de QoS/QoE exigidos pela aplicação de tempo real. Os resultados de simulação mostram a eficácia do esquema proposto em comparação a técnica baseada em RSSI.

\section{Introdução}

As VANETs (Vehicular Ad Hoc Networks) são redes onde os nós móveis são veículos com conexão sem fio [GLASS et al. 2017] que se movem frequentemente entre as áreas de cobertura dos pontos de acesso (APs)/estações base (BSs) de diferentes tecnologias de comunicação sem fio (ex. LTE, 802.11p e 5G), o que requer que suas conexões e sessões de dados sejam transferidas entre tais redes para dar continuidade a aplicação do usuário, processo este denotado como handoff vertical. As VANETs proveem suporte as cidades 
inteligentes e possibilitam que os passageiros de veículos inteligentes possam usufruir de aplicações de entretenimento.

A alta mobilidade e heterogeneidade das tecnologias de acesso à rádio (RATs) das redes veiculares torna o gerenciamento do handoff uma tarefa desafiadora, especialmente quando envolve usuários com aplicações de tempo real. Neste aspecto, o paradigma de Redes Definidas por Software (SDN) tem sido considerado para endereçar esse desafio, trazendo flexibilidade no gerenciamento das VANETS [AL-HEETY et al. 2020] através da separação dos planos de dados e controle, visão da rede logicamente centralizada e inserção de programabilidade na rede [AMIN et al. 2018]. Por exemplo, em cenário de handoff, o SDN possibilita, através de regras definidas no controlador central, que as sessões de dados dos veículos sejam encaminhadas para a melhor estação base/ponto de acesso que atende o veículo no momento, tornando o comportamento da rede programável, facilitando a sua adaptação de acordo com as alterações no ambiente das VANETS.

Abordagens sobre handoff para VANETs têm se mostrado incapazes de gerenciar a transferência de conexão dos veículos entre tecnologias sem fio diferentes (handoff vertical), especialmente em garantir QoS (Qualidade de Serviço) e QoE (Qualidade de Experiência) para aplicações de tempo real [Smida et al. 2018][Goudarzi et al. 2018] [Duo et al. 2018]. A falta de uma estrutura de gerenciamento que viabilize a programabilidade da rede associada à ausência de um processo de tomada de decisão de handoff rápido e enxuto envolvendo múltiplas informações contextuais (ex. potência de sinal do ponto de acesso, velocidade e posição dos veículos) são fatores que contribuem para a ineficiência das técnicas atuais.

Neste sentido, este artigo propõe um arcabouço para prover o handoff transparente definido por software em uma VANET heterogênea e QoS e QoE para aplicações de tempo real. O arcabouço possui um agente baseado em AHP (Analytic Hierarchy Process) responsável pelas decisões de handoff e utiliza o Received Signal Strength Indication (RSSI), a velocidade e a posição dos veículos como informações de contexto. Além disso, a técnica de multihoming compõe a proposta com o intuito de assegurar que o handoff vertical ocorra sem causar intermitências na conexão do veículo com a infraestrutura de acesso sem fio. O AHP possibilita tomadas de decisões rápidas e eficientes em situações com múltiplos parâmetros de entrada [Saaty 1988] e o SDN simplifica o gerenciamento da rede, especialmente durante as transferências de conexão. $\mathrm{O}$ arcabouço proposto foi avaliado via simulador OMNET++, considerando aplicações de tempo real (VoIP e streaming de vídeo) e cenários com veículo em diferentes velocidades, onde métricas de QoS (atraso fim a fim e perda de pacotes) e QoE (Peak signal-to-noise ratio, PSNR) foram analisadas. Os resultados mostraram a superioridade da proposta em até $99 \%$ e $64 \%$ nas métricas de QoS e QoE, respectivamente, quando comparada à abordagem baseada em RSSI.

O restante deste artigo está estruturado da seguinte forma: A Seção 2 discute os trabalhos relacionados. A Seção 3 apresenta o mecanismo proposto para handoff em redes veiculares heterogêneas. A Seção 4 disserta sobre os resultados obtidos nos experimentos com simulação. Por fim, a Seção 5 apresenta as considerações finais. 


\section{Trabalhos Relacionados}

Diferentes trabalhos têm endereçados os desafios do processo de handoff em redes veiculares. Por exemplo, em [Medeiros et al. 2018] os autores apresentam uma estratégia baseada na taxa de entrega de pacotes (TEP), mean opinion score (MOS) preditivo e RSRP (Reference Signal Received Power) para mitigar os impactos do handoff na transmissão de vídeo em tempo real. A técnica AHP foi usada na tomada de decisão, definindo diferentes níveis de importância aos parâmetros. Entretanto, a estratégia é aplicada a cenários de handoff horizontal (transferência entre pontos de acesso da mesma tecnologia) com células de coberturas diferentes, o que limita o usuário a uma tecnologia de comunicação sem fio. Além disso, o arcabouço proposto não viabiliza a programabilidade da rede e não garante o handoff transparente, já que o usuário não dispõe da recepção simultânea de dados por diferentes conexões durante o procedimento.

Já [Smida et al. 2018] propõe um mecanismo que utiliza a predição da qualidade de enlace entre a Road Side Unit (RSU) e o veículo para decidir sobre o handoff vertical. A predição considera a qualidade do enlance das RSUs e os parâmetros de QoE da aplicação. A proposta busca diminuir o tempo de handoff e melhorar a QoE do vídeo, minimizando o tempo de interrupção do streaming, durante o procedimento. Contudo, ela não impede que interrupções ocorram, o que é o desejável e que poderia ser alcançável com o uso da técnica de multihoming, por exemplo.

Com o intuito de prover comunicação contínua entre o veículo e a infraestrutura, os autores em [Ghosh et al. 2015] propõem um esquema de handoff via abordagem de cobertura probabilística. A tomada de decisão de handoff se baseia em fatores como o tempo em que o handoff deve ocorrer (TBVH), o tempo que nó permanece na rede (NDT), calculados probabilisticamente, e a velocidade do veículo na tomada de decisão. Embora TBVH e NDT sejam importantes, não usar informações de contexto da rede como a potência de sinal ou qualidade do enlace, pode comprometer a eficiência do mecanismo e degradar a aplicação, pois elas influenciam a QoS (ex. atraso fim a fim, jitter, taxa de dados) das aplicações. Além disso, a proposta se limita a handoff horizontal.

Em [Skondras et al. 2017], um esquema de handoff vertical para otimizar o gerenciamento da mobilidade dos veículos para sistemas VCC (Vehicular Cloud Computing) é proposto. Os autores assumem um cenário com redes LTE (Long Term Evolution) e IEEE 802.11p e usam o RSSI, a velocidade dos veículos, o atraso fim a fim, o jitter, a taxa de dados enviados/recebidos e a perda de pacotes para apoiar as decisões de disparo de handoff, buscando reduzir o custo das operações, melhorar o gerenciamento de mobilidade do usuário e prover melhor conexão aos veículos. Embora o trabalho vise oferecer a melhor conexão, o tráfego na rede ainda é engessado, onde o caminho que o fluxo de dados pode tomar é limitado ao conhecimento de cada comutador, o que poderia sobrecarregar alguns segmentos da rede. $\mathrm{O}$ uso de SDN contornaria esse problema, pois a visão global do controlador possibilitaria a escolha do melhor caminho para os dados trafegarem.

Para fornecer QoS para os serviços móveis em VANETS, [Goudarzi et al. 2018] propõe um mecanismo de handoff vertical que utiliza otimização em biogeografia e cadeias de Markov no agente de decisão. Eles calculam a probabilidade de um nó mudar de célula em um cenário com redes WiMax (Worldwide Interoperability for Microwave Access), 3G (Third Generation) e Wi-Fi. Os autores consideram informações como geolocalização do veículo, largura de banda da rede, latência da rede, perda de pacotes e 
throughput no handoff, mas negligenciam a velocidade do veículo, elemento importante na decisão de transferência de conexão em ambientes altamente dinâmicos.

Visando aumentar o tempo de conectividade do veículo, reduzir a taxa de falha e quantidade de handoffs desnecessários, [Evangeline and Kumaravelu 2017] propõe uma estratégia de handoff vertical para VANETs, cuja tomada de decisão é baseada no tempo de viagem do veículo, estimado através do valor de RSSI das RSUs. A proposta reduz as falhas no handoff, que é importante para o bom desempenho das aplicações VANETs. Entretanto, aumentar o tempo de conectividade dos veículos pode degradar a QoS das aplicações, pois o veículo pode permanecer em conexão de baixa qualidade por mais tempo. Adicionalmente, embora seja voltado para redes heterogêneas, o trabalho não especifica as tecnologias sem fio adotadas na composição do cenário.

Em [Duo et al. 2018] propõe um mecanismo de handoff vertical baseado em SDN. O SDN é adotado para manter a conexão na camada de transporte inalterada durante o handoff em um ambiente com redes IEEE 802.11p e LTE. Embora o uso de SDN facilite o gerenciamento da rede e a tomada de decisão de handoff, informações de contexto como potência do sinal e velocidade do veículo não são levadas em conta. Ademais, garantir a continuidade da conexão na camada de transporte não implica em garantia de continuidade da conexão na camada física da rede, que é a forma recomendada de se obter um handoff eficiente.

Os autores em [Rizvi and Akram 2018] apresentam uma estratégia de handoff baseada na interface X2 do LTE e SDN. A proposta busca diminuir os tempos de preparação para o handoff e de entrega, e manter íntegra a taxa de dados do usuário durante a preparação e conclusão do processo. Todavia, os autores não especificam os parâmetros e o mecanismo utilizados para a tomada de decisão e direcionam a abordagem apenas para redes LTE, não cobrindo a tecnologia IEEE 802.11p, padrão para VANETs.

Para reduzir o custo de sinalização, o atraso de handoff e a taxa de perda de pacotes da aplicação do usuário, [He et al. 2018] propõe um mecanismo de seleção dinâmica de rede que reutiliza parte das tabelas de fluxo dos switches SDN. O mecanismo é embarcado no controlador SDN e utiliza apenas a velocidade do veículo como parâmetro para a tomada de decisão, negligenciando a potência de sinal. Além disso, a proposta é limitada a ambientes com rede sem fio homogêneas e, similar as abordagens já apresentadas nesta seção, não aplica o multihoming para assegurar a conexão do usuário durante o handoff.

Aplicando multihoming via Multipath TCP (MPTCP) durante o handoff vertical e a diferenciação de tráfego a nível de quadro, [Rene et al. 2015] busca garantir o QoE de vídeo em VANETs. A diferenciação é usada na seleção da melhor rede para a recepção do vídeo e no descarte dos pacotes menos importantes quando a rede está saturada. A realização do handoff é baseado na janela de congestionamento TCP e não emprega SDN. Já [Singh et al. 2019] combina o MPTCP com SDN para prover handoff vertical com multihoming em VANETs. Nele, o handoff é gerenciado pelo controlador SDN, que é ciente da mobilidade dos veículos e injeta regras de encaminhamento de fluxo nos switches quando o veículo está na iminência de sofrer o handoff. Entretanto, o uso do MPTCP implica em criação de subfluxos TCP, um para cada conexão do veículo, não sendo o TCP indicado para procedimentos de handoff, pois ele quebra a janela de congestionamento. 
A Tabela 1 resume o comparativo entre as propostas encontradas na literatura e este trabalho.

Tabela 1. Comparação dos trabalhos relacionados

\begin{tabular}{|c|c|c|c|c|c|c|}
\hline Proposta & Parâmetros & $\begin{array}{l}\text { Inteligên- } \\
\text { cia }\end{array}$ & SDN & $\begin{array}{l}\text { Multiho- } \\
\text { ming }\end{array}$ & $\begin{array}{l}\text { Cenário } \\
\text { Hete- } \\
\text { rogêneo } \\
\end{array}$ & $\begin{array}{l}\text { Técnica } \\
\text { de } \\
\text { Avaliação }\end{array}$ \\
\hline $\begin{array}{l}\text { [Medeiros et } \\
\text { al. 2018] }\end{array}$ & $\begin{array}{lll}\text { TEP, MOS } & \\
\text { RSRP } & & \end{array}$ & AHP & $x$ & $x$ & $x$ & Simulação \\
\hline $\begin{array}{l}\text { [Smida et al. } \\
\text { 2018] }\end{array}$ & $\begin{array}{l}\text { Qualidade de en- } \\
\text { lace e parâmetros } \\
\text { de QoE }\end{array}$ & $\begin{array}{l}\text { Baseada } \\
\text { em função } \\
\text { matemática }\end{array}$ & $x$ & $x$ & $x$ & Simulação \\
\hline $\begin{array}{l}\text { [Ghosh et al. } \\
\text { 2015] }\end{array}$ & $\begin{array}{l}\text { TBVH, NDT e } \\
\text { velocidade do } \\
\text { veículo }\end{array}$ & $\begin{array}{l}\text { Modelo ma- } \\
\text { temático }\end{array}$ & $x$ & $x$ & $x$ & $\begin{array}{l}\text { Simulação e } \\
\text { Testbed }\end{array}$ \\
\hline $\begin{array}{l}\text { [Skondras et } \\
\text { al. 2017] }\end{array}$ & $\begin{array}{l}\text { RSSI, velocidade } \\
\text { do veículo e QoS }\end{array}$ & $\begin{array}{l}\text { Baseada } \\
\text { em função } \\
\text { matemática }\end{array}$ & $x$ & $x$ & $\begin{array}{l}\text { LTE } \quad \mathrm{e} \\
\text { IEEE } \\
802.11 \mathrm{p}\end{array}$ & $\begin{array}{l}\text { Modelo ma- } \\
\text { temático }\end{array}$ \\
\hline $\begin{array}{l}\text { [Goudarzi et } \\
\text { al. 2018] }\end{array}$ & $\begin{array}{l}\text { Localização do } \\
\text { veículo e serviços } \\
\text { de QoS }\end{array}$ & $\begin{array}{l}\text { Baseada } \\
\text { em função } \\
\text { matemática }\end{array}$ & $x$ & $x$ & $\begin{array}{l}\text { WiMAX, } \\
\text { 3G e Wi-Fi }\end{array}$ & $\begin{array}{l}\text { Modelo ma- } \\
\text { temático }\end{array}$ \\
\hline $\begin{array}{l}\text { [Evangeline } \\
\text { and } \mathrm{Ku}- \\
\text { maravelu } \\
\text { 2017] }\end{array}$ & RSSI & $\begin{array}{l}\text { Baseada } \\
\text { em função } \\
\text { matemática }\end{array}$ & $x$ & $x$ & $x$ & $\begin{array}{l}\text { Sem } \\
\text { descrição }\end{array}$ \\
\hline $\begin{array}{l}\text { [Duo et al. } \\
2018]\end{array}$ & Sem descrição & $\begin{array}{l}\text { Sem } \\
\text { descrição }\end{array}$ & $V$ & $x$ & $\begin{array}{ll}\text { LTE } & \mathrm{e} \\
\text { IEEE } & \\
802.11 \mathrm{p} & \\
\end{array}$ & Simulação \\
\hline $\begin{array}{l}\text { Rizvi and } \\
\text { Akram 2018] }\end{array}$ & Sem descrição & $\begin{array}{l}\text { Sem } \\
\text { descrição }\end{array}$ & $\checkmark$ & $x$ & $x$ & Simulação \\
\hline $\begin{array}{lll}{[\mathrm{He} \text { et al. }} \\
2018]\end{array}$ & $\begin{array}{l}\text { Velocidade do } \\
\text { veículo }\end{array}$ & PMIPv6 & $\nu$ & $x$ & $x$ & Emulação \\
\hline $\begin{array}{l}\text { [Rene et al. } \\
\text { 2015] }\end{array}$ & Área de cobertura & $x$ & $x$ & $V$ & $\begin{array}{l}\text { IEEE } \\
802.11 \mathrm{p} \text { e } \\
\text { Rede de } \\
\text { Satélites } \\
\end{array}$ & Simulação \\
\hline $\begin{array}{l}\text { [Sing et al. } \\
\text { 2019] }\end{array}$ & Sem descrição & $x$ & $V$ & $\checkmark$ & $\begin{array}{l}\text { IEEE } \\
802.11 \mathrm{p} \quad \mathrm{e} \\
\text { Wi-Fi }\end{array}$ & Emulação \\
\hline Este Trabalho & $\begin{array}{l}\text { RSSI, velocidade } \\
\text { e posição dos } \\
\text { veículos }\end{array}$ & AHP & $V$ & $\checkmark$ & $\begin{array}{l}\text { IEEE } \\
802.11 \mathrm{p} \quad \mathrm{e} \\
\text { LTE }\end{array}$ & Smulação \\
\hline
\end{tabular}

Para suprir as lacunas apresentadas, este artigo apresenta um mecanismo de handoff vertical para redes veiculares, que: (1) utiliza SDN para simplificar o gerenciamento da rede e dar suporte às transferências de conexão, o handoff, (2) adota a técnica de mul- 
tihoming para assegurar que o handoff ocorra sem intermitências na conexão do veículo, (3) considera informações de contexto da rede (RSSI) e do veículo (velocidade e posição) em (4) um mecanismo enxuto e rápido para a tomada de decisão, o AHP. Adicionalmente, avalia a proposta em termos de QoS e QoE considerando aplicações de tempo real (VoIP e streaming de vídeo) e conectividade via rede celular e IEEE 802.11p.

\section{Mecanismo de Handoff Veicular}

Esta seção descreve o arcabouço para handoff vertical em VANETS, que busca prover handoff transparente com garantia dos requisitos de QoS das aplicações VoIP e streaming de vídeo, e QoE do streaming.

\subsection{Estrutura em Camadas}

O arcabouço proposto possui três camadas, como ilustrado na Figura 1a. A camada de plano de dados veicular comporta os veículos que solicitam os serviços oferecidos pela nuvem e sofrem o handoff entre os pontos de conexão. A camada de plano de dados da infraestrutura é constituída pela infraestrutura de rede (ex. switches openflow, BSs e APs), que provê conexão sem fio para os veículos com a nuvem da operadora e encaminham os seus dados. Por fim, na camada de controle e serviços estão presentes o servidor de aplicações e o controlador SDN. Este último é responsável pela: (1) injeção de regras OpenFlow (OF) nos dispositivos openflow, (2) tomada de decisão de handoff, de acordo com as informações recebidas dos veículos, e (3) decisão sobre aplicação do multihoming durante o handoff. Cada veículo está equipado com interfaces de rede celular (ex. LTE) e IEEE 802.11p, e consomem serviços VoIP e streaming de vídeo da nuvem. A conexão da nuvem com o plano de dados da infraestrutura é realizada através do roteador openflow denotado como Roteador Intermediário OpenFlow (RIOF).

\subsection{Funcionamento do Arcabouço e Multihoming via SDN}

A Figura $1 b$ apresenta a sinalização entre os elementos do arcabouço, ilustrados na Figura 1a. Dado que o veículo possui uma conexão com um ponto de acesso IEEE 802.11p, o mesmo mede o RSSI continuamente. Quando o valor de RSSI fica abaixo de um limiar, o veículo sinaliza o controlador central sobre a baixa intensidade de sinal recebida. Neste trabalho se adotou um limiar de $-65 \mathrm{dBm}$ para que as aplicações VoIP e streaming de vídeo possam funcionar satisfatoriamente [Ben-Mubarak et al. 2013]; [Mahardhika et al. 2012]; [Zhioua et al. 2012]. A notificação do veículo consiste no envio de um pacote com os parâmetros de decisão (valores atuais de RSSI recebidos de sua RSU e das RSUs vizinhas, valor da velocidade (em $\mathrm{m} / \mathrm{s}$ ) e coordenadas de sua posição atual) para o sistema de decisão, localizado no controlador, e indica que o terminal final terá as suas aplicações degradadas em razão da baixa intensidade do sinal. O Agente de Informação é quem agrupa as informações a serem enviadas para o controlador. Neste trabalho, assume-se que o carro contém um sistema de registro de localização tal como o GPS (Global Position System).

No controlador, o Gerenciador de Handoff extrai as informações da mensagem com os parametros de decisão e as encaminha para o Agente Inteligente (descrito na Seção 3.3). Quando o controlador constata que o valor do RSSI está abaixo do limiar definido, ele verifica os valores de RSSI das RSUs próximas ao veículo e cruza com a posição e velocidade do nó, para definir a RSU com o melhor sinal oferecido. O controlador utiliza 


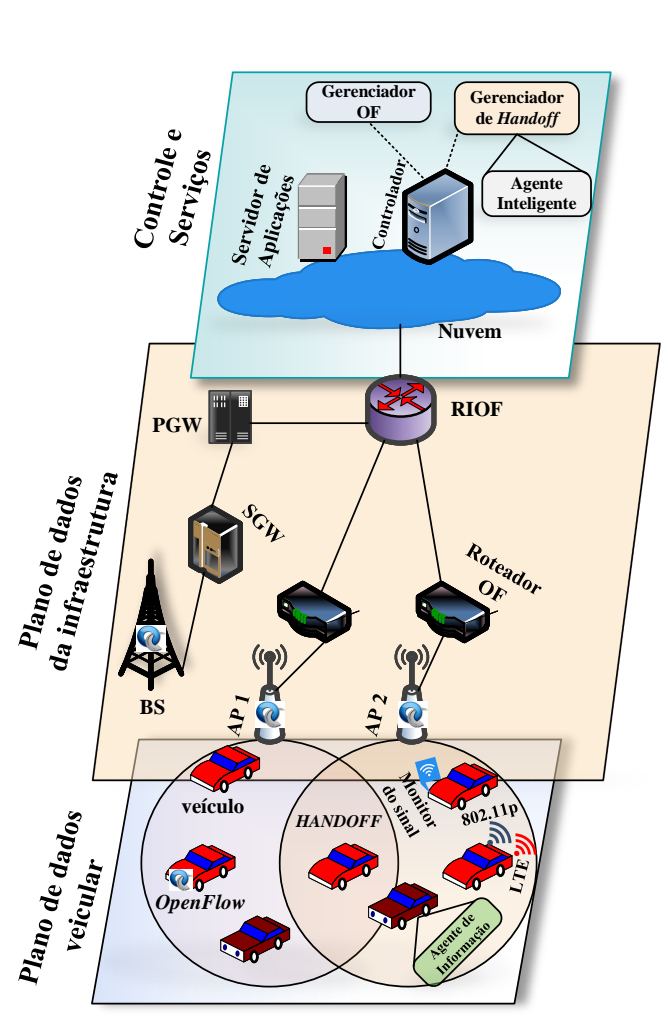

(a)

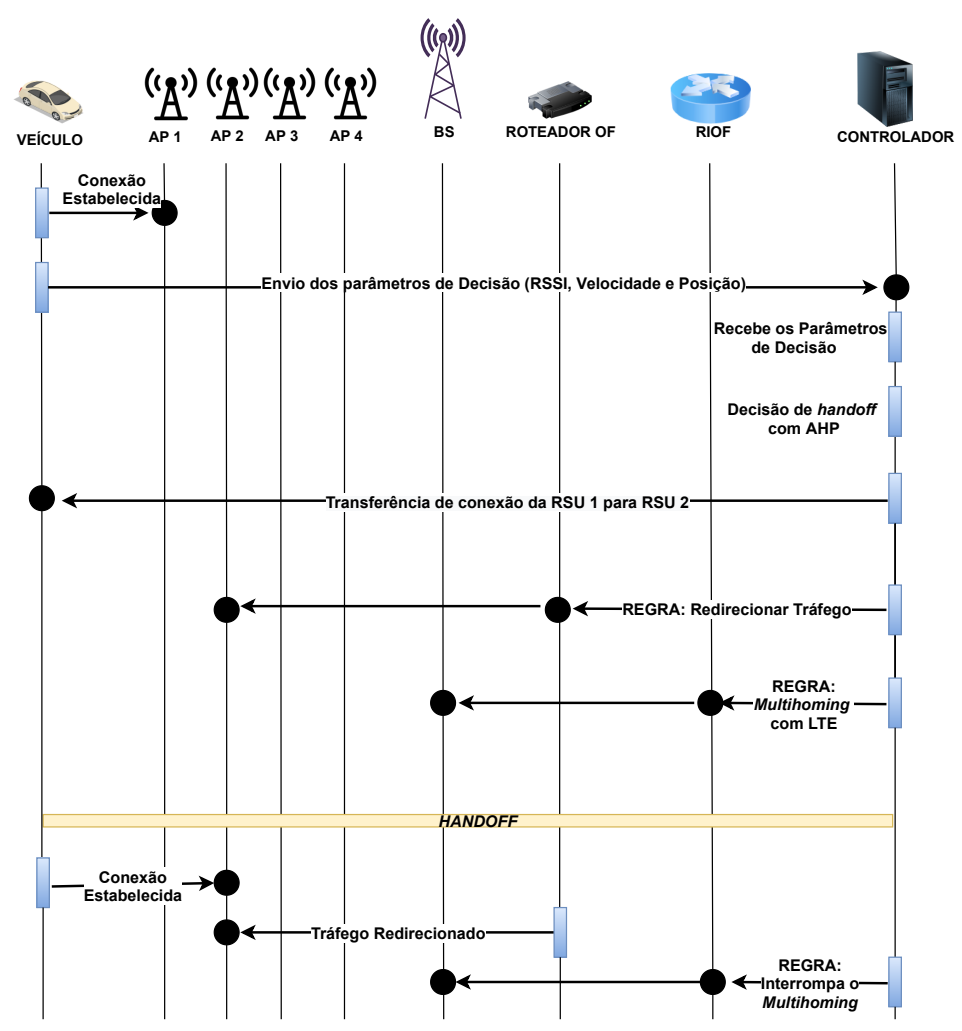

(b)

Figura 1. (a) Estrutura do Arcabouço (b) Sinalização entre os Elementos

as informações das coordenadas do veículo para calcular a distância dele até a RSU mais próxima. Baseado nas RSSIs das RSUs vizinhas e velocidade e posição do veículo, o controlador decide para qual RSU o handoff será feito.

Após a decisão de qual RSU irá receber a conexão do veículo, o controlador envia uma regra para os os roteadores OF (OpenFlow) contendo o número da porta para onde o fluxo de dados será redirecionado em cada um deles. O controlador se comunica com os roteadores via protocolo OpenFlow. A regra injetada nos roteadores fará com que eles encaminhem o fluxo de dados até a RSU onde o veículo se conectará. O Gerenciador OF, situado no controlador, é o responsável por estabelecer a comunicação OpenFlow com os roteadores OF, criar e disparar as regras para estes dispositivos. É importante ressaltar que os gerenciadores Gerenciador OF e Gerenciador de Handoff compõem a camada de aplicação do controlador SDN.

Com a decisão de que o handoff ocorrerá, o controlador sinaliza ao RIOF via protocolo OpenFlow sobre a execução do multihoming com a rede LTE. O controlador cria uma regra específica e a injeta no RIOF. Nesta regra é identificado o número de porta de acesso à rede LTE e a ação de encaminhamento dos pacotes das aplicações VoIP e do streaming de vídeo através desta porta. Desse modo, enquanto o handoff está ocorrendo e os roteadores estão se articulando para redirecionar o tráfego para a RSU de destino do host na rede IEEE 802.11p, o veículo passa a receber o fluxo de dados pela sua interface LTE. É importante frisar que essa regra do multihoming é restrita ao RIOF da rede. 
Quando o handoff é finalizado e o caminho dos pacotes até a nova RSU do veículo na rede IEEE 802.11p encontra-se concluído, o controlador define uma regra para o RIOF para que o multihoming seja interrompido. A nova regra indica que o dispositivo deve interromper o encaminhamento de pacotes VoIP e de streaming para a rede LTE, e manter o encaminhamento desses pacotes para a porta de acesso à rede IEEE 802.11p, finalizando, assim, o ciclo do handoff.

\subsection{Agente Inteligente}

O agente inteligente responsável por avaliar os parâmetros recebidos do veículo e decidir sobre o handoff reside no controlador da rede. Ele utiliza a técnica de AHP [Saaty 1988] para definir os pesos dos parâmetros usados na tomada de decisão. Para prover uma decisão eficiente e instantânea do disparo do handoff, o custo do procedimento foi utilizado, o qual é definido na Equação 1. Ele permite comparar o custo do veículo continuar na rede atual com o de handoff. Caso o custo de handoff seja menor que o custo da rede atual, o controlador decide que a conexão do nó móvel deve ser transferida para a rede candidata. Este custo utiliza os três parâmetros recebidos do veículo, normalizados através da técnica min-max [Hann and Kamber 2000]. O uso de três parâmetros busca dar mais robustez à tomada de decisão, evitando situações de ping-pong quando apenas a RSSI é empregada.

$$
C t H=\left(p_{R} * R_{n}\right)+\left(p_{V} * V_{n}\right)+\left(p_{D} * D_{n}\right)
$$

Na Equação $1, R_{n}$ representa o RSSI das RSUs e é o elemento principal na tomada de decisão do handoff, pois denota a força de sinal que o veículo está recebendo e é diretamente proporcional ao desempenho da execução de aplicações (quanto maior o RSSI, melhor será o funcionamento das aplicações); $V_{n}$ indica a velocidade do veículo, sendo um elemento indispensável na tomada de decisão do handoff em redes veiculares; $D_{n}$ retrata a distância do veículo para a RSU vizinha. Se a distância entre o veículo e a sua RSU atual aumenta em razão da mobilidade dele, ao passo que a distância dele com a RSU vizinha diminui, o controlador poderá optar por realizar o handoff. Além disso, o custo de handoff utiliza pesos definidos pelo AHP para os parâmetros, onde $p_{R}, p_{V}$ e $p_{D}$ são relacionados a RSSI, velocidade e distância, respectivamente.

Para definir os pesos através do AHP, comparações em pares com os três parâmetros foram realizadas, assinalando o grau de importância de um parâmetro em relação ao outro. A Tabela 2 apresenta os resultados dos julgamentos feitos. Em seguida, foi feita a verificação da consistência dos julgamentos através do cálculo da razão de consistência (CR) [Saaty 1988], cujo valor alcançado foi de 0,004 , validando os pesos 0,648 , 0,230 e 0,230 para RSSI, velocidade e distância, respectivamente. Combinando o funcionamento do arcabouço e do agente inteligente, o algoritmo 1 resume as tomadas de decisão durante o processo de handoff vertical, nos lados dos veículos e da rede.

Tabela 2. Tabela de Decisão

\begin{tabular}{|c|c|c|c|}
\hline & $\boldsymbol{R}$ & $\boldsymbol{V}$ & $\boldsymbol{D}$ \\
\hline $\boldsymbol{R}$ & 1 & 3 & 5 \\
\hline $\boldsymbol{V}$ & $\frac{1}{3}$ & 1 & 2 \\
\hline $\boldsymbol{D}$ & $\frac{1}{5}$ & $\frac{1}{2}$ & 1 \\
\hline
\end{tabular}




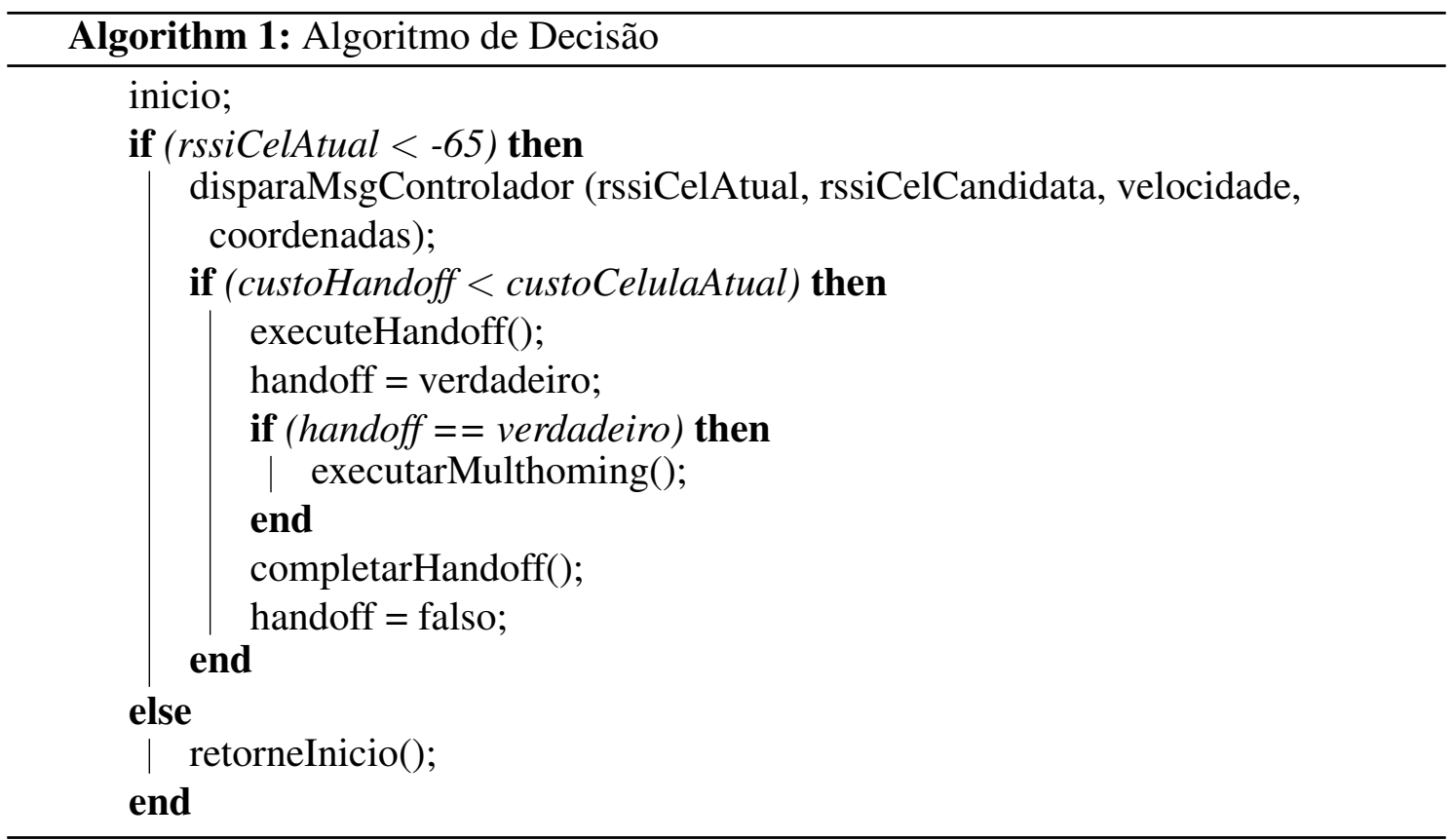

\section{Avaliação}

Para avaliar a proposta, adotou-se o simulador OMNeT++ (versão 5.1), a estrutura VEINS [Sommer et al. 2010] e o SimuLTE [Virdis et al. 2015] para simular a tecnologia IEEE 802.11p) e a arquitetura do cenário LTE, respectivamente. O cenário de simulação consiste em um plano com área de 2200m X 900m, onde 31 veículos equipados com interfaces LTE e IEEE 802.11p se locomovem de forma linear, com velocidades de $15 \mathrm{~m} / \mathrm{s}$, $20 \mathrm{~m} / \mathrm{s}$ e $30 \mathrm{~m} / \mathrm{s}$ em cada caso analisado [Duo et al. 2018]. Além disso, 1 EnodeB, 4 RSUs, 4 roteadores openflow, 1 controlador SDN e um servidor de aplicações (VoIP e streaming de vídeo) compõe o cenário. As áreas de cobertura das quatro RSUs se intersectam duas a duas, ou seja, RSU1 com a RSU2, RSU2 com a RSU3 e a RSU3 com a RSU4, e a eNodeB cobre toda a área do plano. A forma de disposição das células e a seleção de mobilidade linear buscou fazer com que os veículos percorressem as diferentes células durante o movimento, desencadeando handoffs. Os pontos dos gráficos a seguir foram obtidos através de 30 rodadas de simulação de 120s de duração cada, e são expressos com nível de confiança de $95 \%$. A Tabela 3 resume os parâmetros de simulação.

O mecanismo proposto foi avaliado em termos de métricas de QoS (atraso fim a fim e perda de pacotes) e QoE (Peak Signal-to-Noise Ratio - PSNR), medidas durante a ocorrência dos handoffs e com o usuário do veículo consumindo serviços de VoIP e streaming de vídeo. Os seus resultados foram comparados àqueles obtidos com o uso da abordagem baseada somente no RSSI e sem a programabilidade da rede, denotada como tradicional. Nela, o disparo do handoff e a seleção do ponto de acesso ou BS para onde a conexão será transferida considera apenas a intensidade do sinal (ex. RSSI), onde o primeiro acontece quando o RSSI está abaixo de um dado limiar (ex. $-65 \mathrm{dBm}$ ) e o segundo seleciona a BS/AP com maior valor de RSSI [Ahmed et al. 2013].

A Figura 2 apresenta o atraso fim a fim médio considerando a aplicação VoIP. Nota-se que utilizando o mecanismo proposto, o atraso médio de pacotes ficou abaixo de $0,020 \mathrm{~s}$ e $0,030 \mathrm{~s}$, quando o veículo se move com velocidades iguais a $15 \mathrm{ou} 20 \mathrm{~m} / \mathrm{s}$, e 30 
Tabela 3. Parâmetros de Simulação

\begin{tabular}{c|c}
\hline Parâmetros & Valores \\
\hline Tempo de simulação & $120 \mathrm{~s}$ \\
\hline Quantidade de veículos & 31 \\
\hline Aplicações & VoIP e Streaming de vídeo \\
\hline Velocidade & $15 \mathrm{~m} / \mathrm{s}, 20 \mathrm{~m} / \mathrm{s} \mathrm{e} 30 \mathrm{~m} / \mathrm{s}$ \\
\hline Roteadores & 4 \\
\hline RSUs & 4 \\
\hline eNodeB & 1 \\
\hline Controlador & 1 \\
\hline Modelo de mobilidade & Linear \\
\hline Modelo de propagação & Two-Ray \\
\hline
\end{tabular}

$\mathrm{m} / \mathrm{s}$, respectivamente, ao passo que com a abordagem tradicional, o atraso médio foi de $0,045,0,070 \mathrm{~s}$ e 0,1 para as velocidades de 15,20 e $30 \mathrm{~m} / \mathrm{s}$, respectivamente. Dessa forma, a redução alcançada com o mecanismo proposto foi em média de $62 \%$ (em $15 \mathrm{~m} / \mathrm{s}$ ) e $74 \%$ $(\mathrm{em} 20 \mathrm{~m} / \mathrm{s})$ e de até $74 \%(\mathrm{em} 30 \mathrm{~m} / \mathrm{s})$.

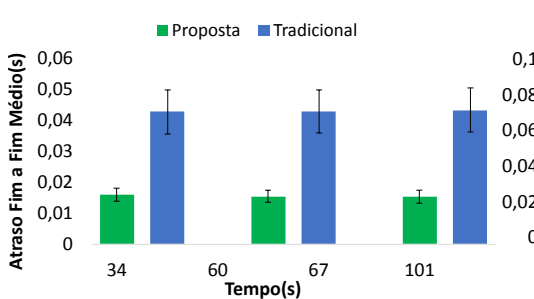

(a)

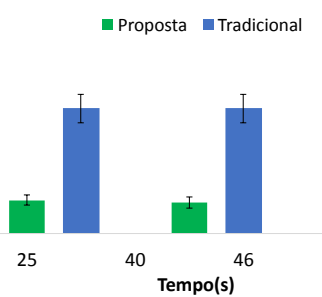

(b)

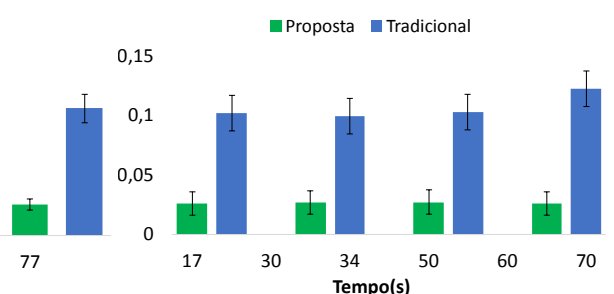

(c)

Figura 2. Atraso fim a fim na aplicação VoIP quando o veículo se movimenta a (a) $15 \mathrm{~m} / \mathrm{s}$, (b) $20 \mathrm{~m} / \mathrm{s}$ e (c) $30 \mathrm{~m} / \mathrm{s}$

O veículo inicia sua conexão no IEEE 802.11 p, mas a partir dos 70 s de simulação, com o nó a 30m/s (Figura 2c), a cobertura da rede IEEE 802.11p é perdida e, em seguida, o veículo estabelece conexão com a rede LTE, provocando uma interrupção momentânea no serviço. Em razão disso, o pico do atraso médio durante o handoff no sistema tradicional foi de $0,12 \mathrm{~s}$. Em contrapartida, no sistema proposto, o controlador da rede dispara a ação de multihoming com o LTE para a camada intermediária antes que o veículo perca a cobertura de sua RSU. Com isso, o serviço não foi interrompido e o atraso médio de pacotes foi de apenas $0,026 \mathrm{~s}$. É importante ressaltar que após o handoff o nó retorna para o IEEE 802.11p.

A decisão precisa, instantânea e eficiente do agente inteligente que reside no controlador SDN e o uso da técnica de multihoming com as tecnologias 802.11p e LTE possibilitaram a redução do atraso fim a fim durante o handoff quando do uso da estratégia proposta. Baseados nas informações de contexto, o agente decisor seleciona a melhor rede para o veículo se conectar, e dispara o multihoming durante o processo de handoff, evitando degradação na conexão do usuário.

A Figura 3 apresenta os resultados de perda de pacotes na aplicação VoIP durante 
os handoffs. Com a abordagem tradicional, a perda média foi acima de 50, 60 e 80 pacotes com o veículo a 15,20 e $30 \mathrm{~m} / \mathrm{s}$, respectivamente. Já com o mecanismo proposto, apenas 1 pacote foi perdido, independente da velocidade do veículo, o que aponta para uma eficiência próxima dos $100 \%$.

Tendo ciência de que a conexão do veículo está prestes a se degradar, o agente decisor determina que o handoff deve ser executado e seleciona a melhor RSU para a transferência da conexão. O controlador injeta regras SDN nas tabelas de fluxos dos switches SDN, instruindo a execução do procedimento para a RSU selecionada. Simultaneamente, o controlador envia uma regra para o RIOF, notificando-o para a realização do multihoming entre as tecnologias 802.11p e LTE. Deste modo, o mecanismo proposto consegue alcançar essa redução expressiva na perda de pacotes. A partir dos 70 s de simulação, com o veículo a 30m/s (Figura 3c) e usando a abordagem tradicional, tem-se 90 pacotes perdidos. Isso ocorreu devido a perda de conexão do veículo com o seu ponto de acesso IEEE 802.11p, e, posteriormente, ancoramento na rede LTE, ocasionando uma quebra na comunicação V2I. Diferentemente, o arcabouço proposto faz uso do multihoming antecipado entre o IEEE 802.11p e a rede LTE para evitar quebra de conexão sem fio do veículo e assim garantir o bom desempenho da aplicação VoIP no lado do usuário.

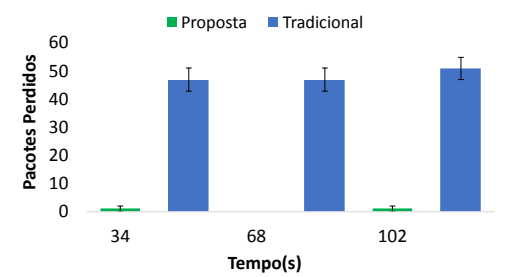

(a)

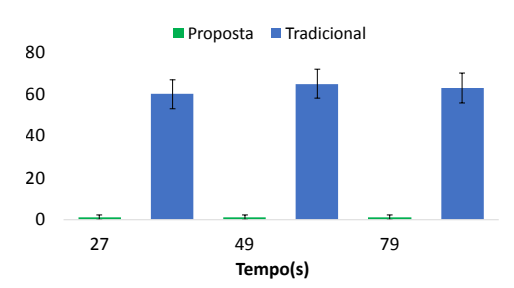

(b)

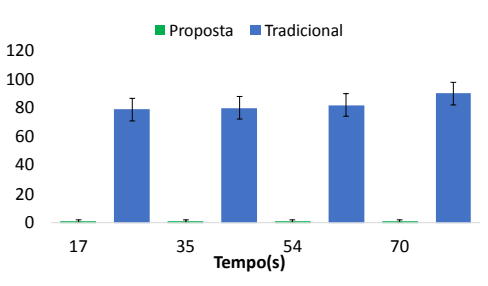

(c)

Figura 3. Perda de pacotes na aplicação VoIP quando o veículo se movimenta a (a) $15 \mathrm{~m} / \mathrm{s}$, (b) $20 \mathrm{~m} / \mathrm{s}$ e (c) $30 \mathrm{~m} / \mathrm{s}$

Em termos de atraso fim a fim no streaming de vídeo (ver Figura 4), nota-se que com a abordagem proposta o atraso médio de pacotes durante o handoff foi de $0,015 \mathrm{~s}$, $0,025 \mathrm{~s}$ e $0,035 \mathrm{~s}$ quando o veículo se move a 15,20 e $30 \mathrm{~m} / \mathrm{s}$, respectivamente. Já com o esquema tradicional, o atraso médio ficou acima dos $0,050 \mathrm{~s}(\mathrm{em} 15 \mathrm{~m} / \mathrm{s}), 0,065 \mathrm{~s}(\mathrm{em} 20$ $\mathrm{m} / \mathrm{s})$ e $0,090 \mathrm{~s}(\mathrm{em} 30 \mathrm{~m} / \mathrm{s})$. Estes valores indicam uma redução de $71 \%$, $63 \%$ e $71 \%$ no atraso médio, alcançada pelo mecanismo proposto nos cenários com velocidade veicular de 15,20 e $30 \mathrm{~m} / \mathrm{s}$, respectivamente.

Analisando a quebra de conexão que ocorre a partir do tempo de simulação 70s, onde o veículo, se movendo a 30m/s (Figura 4c), fica sem cobertura IEEE $802.11 \mathrm{p}$ e se conecta à rede LTE. Com a abordagem tradicional, o processo de desconexão e conexão provoca interrupções no serviço, fazendo com que o pico do atraso fique em $0,12 \mathrm{~s}$. Por outro lado, com o mecanismo proposto, a perda da área de cobertura IEEE 802.11p não ocasiona danos à aplicação, pois o agente centralizado da rede dispara a execução do multihoming com o LTE antes que a cobertura da RSU seja perdida, proativamente. Com isso, o atraso médio no streaming de vídeo ficou em 0,029s.

A Tabela 4 apresenta os resultados da métrica PSNR alcançados pelas abordagens. Com o sistema proposto, o PSNR ficou acima dos $34 \mathrm{~dB}$ para todas as velocidades analisadas. Este é um valor considerado bom na escala de qualidade da métrica 


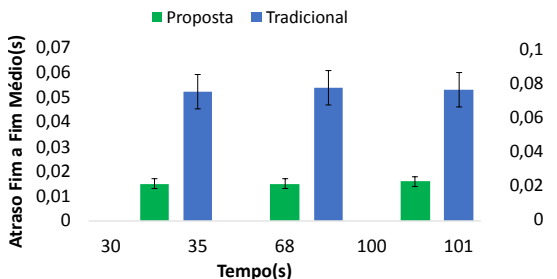

(a)

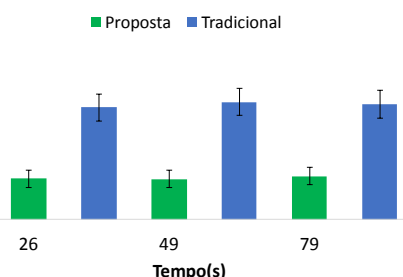

(b)

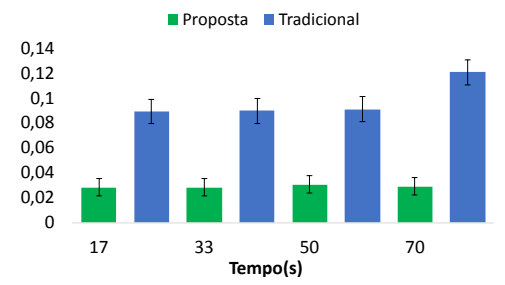

(c)

Figura 4. Atraso fim a fim no streaming de vídeo quando o veículo se movimenta a (a) $15 \mathrm{~m} / \mathrm{s}$, (b) $20 \mathrm{~m} / \mathrm{s}$ e (c) $30 \mathrm{~m} / \mathrm{s}$

[Thung and Raveendran 2009]; [Huynh-Thu and Ghanbari 2012]. Já com a abordagem tradicional, o PSNR ficou abaixo dos $18 \mathrm{~dB}$, valor considerado péssimo, com o veículo se movendo a $15 \mathrm{~m} / \mathrm{s}$, permaneceu em $16 \mathrm{~dB}$ e caiu para $13 \mathrm{~dB}$, quando o veículo se movia a 20 e $30 \mathrm{~m} / \mathrm{s}$, respectivamente. Em termos de ganho no valor de PSNR em relação a abordagem tradicional, o sistema proposto alcançou valores de 53\%, 55\% e $64 \%$ com o veículo se movendo a 15,20 e $30 \mathrm{~m} / \mathrm{s}$, respectivamente.

Tabela 4. PSNR do streaming de vídeo quando o veículo se movimenta a (a) $15 \mathrm{~m} / \mathrm{s}$, (b) $20 \mathrm{~m} / \mathrm{s}$ e (c) $30 \mathrm{~m} / \mathrm{s}$

\begin{tabular}{cccc}
\hline \hline Velocidade & PSNR - Proposta & PSNR - Tradicional & Eficiência da Proposta \\
\hline $15 \mathrm{~m} / \mathrm{s}$ & $37 \mathrm{dBm}$ & $17 \mathrm{dBm}$ & $\mathbf{5 3 \%}$ \\
\hline $20 \mathrm{~m} / \mathrm{s}$ & $36 \mathrm{dBm}$ & $16 \mathrm{dBm}$ & $\mathbf{5 5 \%}$ \\
\hline $30 \mathrm{~m} / \mathrm{s}$ & $35 \mathrm{dBm}$ & $13 \mathrm{dBm}$ & $\mathbf{6 4 \%}$ \\
\hline \hline
\end{tabular}

Com o mecanismo proposto, os valores de PSNR permanecem no intervalo considerado bom porque o veículo passa a receber o vídeo via multihoming durante o handoff. Além disso, a sinergia entre o sistema de decisão com AHP, o que torna a decisão de handoff instantânea e proativa, e o esquema de programabilidade do comportamento da rede com SDN, além da técnica de multihoming, contribui para que o mecanismo proposto obtenha melhor desempenho, comparado a abordagem tradicional, em mitigar os impactos do handoff na QoE do vídeo, no lado do usuário. A abordagem baseada somente na potência de sinal causa intermitências na conexão durante o procedimento de transferência de conexão, degradando os valores de PSNR da aplicação. Já o sistema proposto neste trabalho, garante o handoff transparente para o usuário, assegurando os requisitos de QoE para o bom desempenho da aplicação.

\section{Considerações Finais}

Este artigo apresentou um mecanismo de handoff vertical para redes veiculares com o intuito de assegurar requisitos de QoS e QoE exigidos por aplicações de tempo real. O mecanismo aplicou o AHP nas tomadas de decisões de handoff, levando em consideração os parâmetros RSSI, velocidade e posição dos veículos, como informações de contexto. Além disso, o sistema utilizou o paradigma SDN para viabilizar a programabilidade do plano de controle, particularmente, em relação ao procedimento de handoff e a técnica de multihoming para garantir que o processo de transferência de conexão ocorresse sem 
interrupções. Os resultados obtidos mostraram a eficiência do mecanismo em comparação a abordagem tradicional, onde ganhos de $62 \%$ a $74 \%$ na redução do atraso fim a fim, de até $99 \%$ na redução de perda de pacotes e de até $64 \%$ no PSNR foram obtidos. Como direções futuras de pesquisa, destacam-se o uso de sistemas Fuzzy associado ao AHP para tornar o sistema decisor mais robusto; utilização de informação de carga da rede de acesso para evitar a transferência da conexão do veículo para uma célula sobrecarregada;realizar o balanceamento de carga do tráfego durante o multihoming; e englobar as redes $5 \mathrm{G}$ na composição da proposta.

\subsection{Agradecimento}

Os autores agradecem o apoio da FACEPE (Fundação de Amparo à Ciência e Tecnologia do Estado de Pernambuco), através do processo $n^{\circ}$ IBPG-0792-1.03/17, e ao CNPq (projeto $n^{\circ} \mathbf{4 3 3 1 4 2 / 2 0 1 8 - 9}$ e projeto $n^{\circ} 307053 / 2017-2$ ).

\section{Referências}

Ahmed, A., Boulahia, L. M., and Gaiti, D. (2013). Enabling vertical handover decisions in heterogeneous wireless networks: A state-of-the-art and a classification. IEEE Communications Surveys \& Tutorials, 16(2):776-811.

AL-HEETY, O. S., Zakaria, Z., Ismail, M., Shakir, M. M., Alani, S., and Alsariera, H. (2020). A comprehensive survey: Benefits, services, recent works, challenges, security, and use cases for sdn-vanet. IEEE Access, 8:91028-91047.

AMIN, R., REISSLEIN, M., and SHAH, N. (2018). Hybrid sdn networks: A survey of existing approaches. IEEE Communications Surveys Tutorials, 20(4):3259-3306.

Ben-Mubarak, M. A., Ali, B. M., Noordin, N. K., Ismail, A., and Ng, C. K. (2013). Fuzzy logic based self-adaptive handover algorithm for mobile wimax. Wireless Personal Communications, 71(2):1421-1442.

Duo, R., Wu, C., Yoshinaga, T., and Ji, Y. (2018). Sdn-based handover approach in ieee $802.11 \mathrm{p}$ and lte hybrid vehicular networks. In 2018 IEEE SmartWorld, Ubiquitous Intelligence \& Computing, Advanced \& Trusted Computing, Scalable Computing \& Communications, Cloud \& Big Data Computing, Internet of People and Smart City Innovation (SmartWorld/SCALCOM/UIC/ATC/CBDCom/IOP/SCI), pages 18701875. IEEE.

Evangeline, C. S. and Kumaravelu, V. B. (2017). Decision process for vertical handover in vehicular adhoc networks. In 2017 International conference on Microelectronic Devices, Circuits and Systems (ICMDCS), pages 1-5. IEEE.

Ghosh, A., Paranthaman, V. V., Mapp, G., Gemikonakli, O., and Loo, J. (2015). Enabling seamless v2i communications: toward developing cooperative automotive applications in vanet systems. IEEE Communications Magazine, 53(12):80-86.

GLASS, S., MAHGOUB, I., and RATHOD, M. (2017). Leveraging manet-based cooperative cache discovery techniques in vanets: A survey and analysis. IEEE Communications Surveys Tutorials, 19(4):2640-2661.

Goudarzi, S., Hassan, W. H., Anisi, M. H., Khan, M. K., and Soleymani, S. A. (2018). Intelligent technique for seamless vertical handover in vehicular networks. Mobile Networks and Applications, 23(6):1462-1477. 
Hann, J. and Kamber, M. (2000). Data mining: concepts and techniques.

He, Z., Fu, B., Cao, A., and Yu, J. (2018). A solution for mobility management in software defined vanet. In 2018 IEEE 15th International Conference on Mobile Ad Hoc and Sensor Systems (MASS), pages 553-558. IEEE.

Huynh-Thu, Q. and Ghanbari, M. (2012). The accuracy of psnr in predicting video quality for different video scenes and frame rates. Telecommunication Systems, 49(1):35-48.

Mahardhika, G., Ismail, M., and Mat, K. (2012). Multi-criteria vertical handover decision in heterogeneous network. In 2012 IEEE Symposium on Wireless Technology and Applications (ISWTA), pages 1-4. IEEE.

Medeiros, I., Pacheco, L., Rosário, D., Both, C., Nobre, J., Cerqueira, E., and Granville, L. (2018). Handover ciente de qualidade de experiência e qualidade de serviço para transmissão de vídeo em redes heterogêneas. In Anais do XXIII Workshop de Gerência e Operação de Redes e Serviços. SBC.

Rene, S., Exposito, E., Gineste, M., Alins, J., and Esparza, O. (2015). Multipath tcp architecture for infotainment multimedia applications in vehicular networks. In 2015 IEEE 81st Vehicular Technology Conference (VTC Spring), pages 1-5. IEEE.

Rizvi, H. and Akram, J. (2018). Handover management in 5g software defined network based v2x communication. In 2018 12th International Conference on Open Source Systems and Technologies (ICOSST), pages 22-26. IEEE.

Saaty, T. L. (1988). What is the analytic hierarchy process? In Mathematical models for decision support, pages 109-121. Springer.

Singh, P. K., Sharma, S., Nandi, S. K., and Nandi, S. (2019). Multipath tcp for v2i communication in sdn controlled small cell deployment of smart city. Vehicular communications, 15:1-15.

Skondras, E., Michalas, A., Sgora, A., and Vergados, D. D. (2017). A vertical handover management scheme for vanet cloud computing systems. In 2017 IEEE Symposium on Computers and Communications (ISCC), pages 371-376. IEEE.

Smida, E. B., Fantar, S. G., and Youssef, H. (2018). Link quality and qoe aware predictive vertical handoff mechanism for video streaming in urban vanet. In International Conference on Intelligent Systems Design and Applications, pages 170-181. Springer.

Sommer, C., German, R., and Dressler, F. (2010). Bidirectionally coupled network and road traffic simulation for improved ivc analysis. IEEE Transactions on mobile computing, 10(1):3-15.

Thung, K. and Raveendran, P. (2009). A survey of image quality measures. In 2009 International Conference for Technical Postgraduates (TECHPOS), pages 1-4.

Virdis, A., Stea, G., and Nardini, G. (2015). Simulating lte/lte-advanced networks with simulte. In Simulation and Modeling Methodologies, Technologies and Applications, pages 83-105. Springer.

Zhioua, G. e. m., Labiod, H., Tabbane, N., and Tabbane, S. (2012). An efficient qos based gateway selection algorithm for vanet to lte advanced hybrid cellular network. In Proceedings of the 15th ACM international conference on Modeling, analysis and simulation of wireless and mobile systems, pages 353-356. 